\title{
Evaluation of serum level of biotin and effect of biotin replacement therapy in patients with telegon effluvium
}

\author{
H.H.Sabry ${ }^{1}$, S.H.Abd El Rahman ${ }^{1}$, M.A.El Awady ${ }^{2}$, A.M.Noureldine ${ }^{3}$ and R.A.Salama ${ }^{1}$ \\ ${ }^{1}$ Dermatology, Andrology \& Venereology, Dept., Faculty of Medicine, Benha Univ., Benha, Egypt \\ ${ }^{2}$ Community, Dept., Faculty of Medicine, Benha Univ., Benha, Egypt \\ ${ }^{3}$ Clinical and Chemical Pathology, Dept., Faculty of Medicine, Benha Univ., Benha, Egypt \\ E-mail: rana_ali31@yahoo.com
}

\begin{abstract}
Background: Biotin is a water-solutions vitamin used for five mammalian carboxylases as an important coenzyme. It has a major role in fatty acids, amino acids and glucose metabolism. It is a popular dietary supplement for the treatment of fragile nails and hair loss. Objectives: The present research aimed at evaluating patients with or without biotin deficiency in telegon effluvium and determining after 3 months the impact of biotin replacement treatment. Methods: 130 patients with Télégon Efluvium (group A) and 120 healthy individuals (group B) served as a control group were studied. Methods: Those subjects who had been subjected to biotin treatment three months before the survey and reported additional hair loss reasons were eliminated. All patients were assessed for hair loss by clinical diagnosis before and after replacement treatment (hair pull test and Dermoscopic evaluation). The biotin level of serum was measured using commercially available immunosorbent assay kits. Results: Mean biotin serum levels were lower than controls between the two groups in a group. The impact of biotin replacement treatment has shown that better cases are $51.5 \%$, improved cases are $34.6 \%$ and missing cases $13.8 \%$. Patients with insufficient serum biotin levels were substantially improved, while non-improved cases with optimum blood biotin concentrations were significantly identified. Conclusion: Our results indicate that biotin addition had a substantial impact on hair loss, particularly in individuals with telegon effluvium who had deficiencies or inadequate biotin levels.
\end{abstract}

Key words: Anagen, Biotin, Follicles, Hair loss, Telogen Effluvium.

\section{Introduction}

Hair loss is frequently upsetting and may have a major impact on the quality of life of the patient [1]. There are various kinds of hair loss e.g., male hair loss pattern, female hair loss pattern, alopecia areata, anagenic effluvium and TE [2].

Telogen effluvium is a relatively rapid hair loss of non-scarring, non-inflammatory, diffuse hair induced by physiological or emotional stress. The hair will regenerate after the triggering cause is eliminated [3]. The TE may be acute (less six months) or chronic (beyond six months) [4].

There are several possible triggers involved in the pathophysiology of TE, including as stress, hormone changes and medicines [5].

Biotin is referred to as vitamin $\mathrm{H}(\mathrm{H}$ is hair and skin, German "hair and skin") or vitamin B7. Biotin is a cofactor for carboxylase enzymes which, in order to maintain good skin and hair, are essential for different methods of metabolism engaged in fatty acid syntheses, amino acid branching and gluconeogenesis. Normal blood biotin levels exceed $400 \mathrm{ng} / \mathrm{L}$ (optimal) and 200$400 \mathrm{ng} / \mathrm{L}$ and are termed biotin insufficiency if below 200 ng/L. [6].

Symptoms of biotin deficiency include hair loss, dermatitis (a scaly red eye, nose and mouth rash) and neurological symptoms (a 'biotin deficient face,' such as sadness, fatigue, hallucination, stomach and tingling) [7].

Biotin is a popular dietary supplement for treating fragile nails and hair loss because to its availability, affordability and successful marketing [8].

The objective of this paper was to evaluate individuals with or without TE with biotin insufficiency and to determine the impact of biotin replacement treatment after three months.

\section{Subjects and methods}

\subsection{The study population}

This was a case-control randomized clinical trial. Patients were included in this study were from outpatient clinic of Dermatology, Venereology and Andrology Department of Benha University Hospitals in the period from December 2018 to May 2019 after the approval by Research Committee at Faculty of Medicine, Benha University. This study included 130 patients suffering from TE (Group A) and 120 apparently healthy subjects serving as a control group (Group B) matched for age, sex, and body mass index (BMI). Every subject was informed about the aim of the study and an informed consent was obtained from each individual before sample collection. Subjects who were on biotin therapy 3 months prior to the study and those reporting other causes of hair loss, such as androgenetic alopecia, were excluded from this study. Patients were subjected to full history-taking including; onset, course, and duration of hair loss, stress factors, and history of prescriptions and any other medical disorders. Scalp examination was done to exclude other causes of hair loss. Hair pull test was done to evaluate severity and site of hair loss and dermoscopic examination of the scalp was carried out using a dermoscope (3Gen Dermlite HUD, polarized, 10x, American).

\subsection{ELISA assays of Biotin}

Five $\mathrm{ml}$ of venus blood samples were taken from all patients and controls. Then, under complete aseptic 
conditions, samples were collected in a plain serum separating tube and left to clot for $30 \mathrm{~min}$ at room temperature, centrifuged at $3000 \mathrm{rpm}$ for $10 \mathrm{~min}$ and sera were separated at $-20^{\circ} \mathrm{c}$. Biotin serum levels were measured using a commercially available biotin enzyme-linked immunosorbent assay kit which provided by SHANGHAI KORIAN BIOTECH CO.

\subsection{Statistical Analysis}

The collected data was revised, coded, tabulated and introduced to a PC using Statistical package for Social Science (IBM Corp. Released 2011. IBM SPSS Statistics for Windows, Version 20.0. Armonk, NY: IBM Corp.). Data were presented and suitable analysis was done according to the type of data obtained for each parameter. Student T Test was used to assess the statistical significance of the difference between two study group means. For the comparison of the three groups' means, one way analysis of variance (ANOVA) was used. Chi-Square test was used to examine the relationship between two qualitative variables. Fisher's exact test: was used to examine the relationship between two qualitative variables when the expected count is less than 5 in more than $20 \%$ of cells.
Correlation analysis: To assess the strength of association between two quantitative variables. The correlation coefficient defines the strength and direction of the linear relationship between two variables. $\mathrm{p}$ is significant if $<0.05$ at confidence interval $95 \%$.

\section{Results}

Thirty nine patients (30\%) had positive family history, $2.3 \%$ were smokers, $11.5 \%$ were athletes, $28.5 \%$ had psychological symptoms (stress), 9.2\% had recurrent infection, $6.9 \%$ were pregnant and $16.2 \%$ were lactating Table (1).

Mean serum levels of biotin among cases' group were lower than controls with a significant difference between both groups ( $\mathrm{p}$-value < 0.001) Table (2).

According to effect of biotin replacement therapy, improved cases were $51.5 \%$, non- improved cases were $34.6 \%$ and missing cases during follow up were $13.8 \%$. Improved cases were significantly detected in those with deficient serum biotin levels; however nonimproved cases were significantly detected in those with optimal serum biotin levels $(\mathrm{p}$-value $=0.001)$ Table (3\&4).

Table (1) Personal history of cases' group.

\begin{tabular}{lcc}
\hline & Cases' group & \\
& $\mathbf{N}(\mathbf{1 3 0})$ & $\%$ \\
\hline Family history of telegon effluvium & & \\
+ ve & 39 & 30.0 \\
- ve & 91 & 70.0 \\
Athletes & 15 & 11.5 \\
Alcoholics & 0 & 0.0 \\
Smokers & 3 & 2.3 \\
Psychological symptoms (stress) & 37 & 28.5 \\
Recurrent infection & 12 & 9.2 \\
Pregnancy & 9 & 6.9 \\
Lactation & 21 & 16.2 \\
\hline
\end{tabular}

Table (2) Serum levels of biotin in this study.

\begin{tabular}{lcccccc}
\hline & \multicolumn{2}{c}{ Cases' group } & \multicolumn{2}{c}{ Control group } & \multirow{2}{*}{ Student t test } & \multirow{2}{*}{ P -value } \\
& Mean & $*$ SD & Mean & $*$ SD & \multirow{2}{*}{ Stud } \\
\hline Serum biotin $(\mathbf{n g} / \mathbf{m l})$ & 0.27 & 0.23 & 0.72 & 0.26 & 14.51 & $<0.001^{* *}$ \\
\hline
\end{tabular}

Table (3) Association of serum biotin levels to effect on patient after replacement therapy.

\begin{tabular}{|c|c|c|c|c|c|}
\hline \multirow[t]{2}{*}{ Effect (130) } & \multirow[t]{2}{*}{$\mathrm{N}(\%)(112)$} & \multicolumn{2}{|c|}{ Serum biotin } & \multirow[t]{2}{*}{ Student t test } & \multirow[t]{2}{*}{$P$ - value } \\
\hline & & Mean & SD & & \\
\hline \multicolumn{6}{|l|}{ Missing 18 (13.8\%) } \\
\hline Improved 67(51.5\%) & $67(59.8)$ & 0.23 & 0.21 & 3.38 & $0.001 *$ \\
\hline Not improved $45(34.6 \%)$ & $45(40.2)$ & 0.38 & 0.25 & & \\
\hline
\end{tabular}


Table (4) Effect of biotin replacement therapy according to biotin sublevels (deficient, sub-optimal and optimal).

\begin{tabular}{|c|c|c|c|c|c|c|c|c|}
\hline & \multicolumn{2}{|c|}{ Deficient (73) } & \multicolumn{2}{|c|}{ Suboptimal (30) } & \multicolumn{2}{|c|}{ Optimal (27) } & \multirow{2}{*}{$\begin{array}{c}\text { Statistical } \\
\text { test }\end{array}$} & \multirow[t]{2}{*}{$P$ - value } \\
\hline & No & $\%$ & No & $\%$ & No & $\%$ & & \\
\hline & & & & & & & $* \mathrm{FET}=45.3$ & \\
\hline Missed & 12 & 16.4 & 6 & 20.0 & 0 & 0.0 & & \\
\hline Improved & 52 & 71.2 & 9 & 30.0 & 6 & 22.2 & & \\
\hline Not-improved & 9 & 12.3 & 15 & 50.0 & 21 & 77.8 & & $<0.001 * *$ \\
\hline
\end{tabular}

\section{Discussion}

Telogen effluvium is a type of unscratching alopecia with widespread, frequently acute hair loss [9[. It may occur in individuals of all ages, genders and races. A significant proportion of individuals at some time have a TE episode. Women are more likely to suffer this illness due to hormonal changes in postpartum [10].

Biotin is a co-enzyme for carboxylase enzymes and is also known as vitamin B7 or vitamin $\mathrm{H}$. The metabolism of glucose, ramificated amino acids and fatty acids is important [6]. The inadequate dietary consumption of biotin, interactions between medicine and vitamins and possibly increased biotin catabolism during pregnancy and smokers may induce biotin insufficiency. Symptoms of its deficiency include hair loss, scaly sores, erythematosus and eczema [11].

The objective of this research was to evaluate patients with or without biotin deficiency in TE and to determine after 3 months the impact of biotin replacement treatment.

According to the personal history of this research, the family has a good $30 \%$ history, smokers $2.3 \%$, athletes $11.5 \%$, psychological symptoms $28.5 \%$ (stress), repeated infections $9.2 \%$, pregnancies $6.9 \%$ and lactates $16.2 \%$. We observed that $50 \%$ of the patients had positive family records, similar to the Abdel Rahman et al. [12] research. Smokers were also $6.7 \%$, athletes were $13.3 \%$ and recurring illnesses were $6.7 \%$. $20 \%$ of the patients were pregnant, with $30 \%$ having psychological symptoms, including lower selfesteem, sadness, and anxiety. In relation to the links between psycho-emotional stress and hair loss, there are a number of degrees of interaction. One was acute or chronic stress as a main activator of TE or secondary stress as a consequence of a previous hair loss. This may also help to sustain or exacerbate hair loss and lead to a self-preserving vicious cycle [13].

The mean blood levels of biotin were lower in the current research than controls with a significant difference between the two groups. Our understanding suggests that just one research [12] evaluated the serum biotin level in TE patients, as opposed to ours. Biotin serum concentration was optimum $(>0.4 \mathrm{ng} / \mathrm{mL})$ in both groups. There were greater serum levels of biotin with no significant differences between the two groups. In androgenic alopecia (14), two investigations were conducted at blood biotin level one and the other in premature canities ( $<20$ years of age) (15). The findings were similar to ours, as the average blood level of biotin in patients compared to control was considerably lower.

$51.5 \%$ of patients exhibited an increase in the efficacy of biotin replacement treatment in this research, whereas $34.6 \%$ showed no change. Those with lower blood biotin levels were substantially better; however, cases with higher serum biotin levels with higher serum levels were not significantly improved. Patel et al. [8] observed a substantial impact of biotin supplementation on 18 individuals suffering from underlying disease of inadequate hair or nail development. Also, Almohanna et al. [16] demonstrated that 3-5 mg biotin daily in children with incombable hair syndrome may enhance hair health after 3-4 months.

\section{Conclusion}

Based on the findings of this research, biotin supplementation has shown a substantial impact on hair loss in TE patients, notably in those patients with either deficiency or under-optimum biotin levels.

\section{Recommendations}

In view of its limitations, the findings of the current research should be interpreted since the present study included a very small sample. Further research are necessary to analyse the tissue expression of biotin in vitro in acute and chronic TE, examine the accurate mechanisms by which biotin contributes to TE pathogenesis, and evaluate the roles of biotin in TE therapy.

\section{References}

[1] N.Hunt ,S.McHale. The psychological impact of alopecia . BMJ. vol. (3317522) ,pp.951-953 ,2005.

[2] J.Vary. Selected Disorders of Skin AppendagesAcne, Alopecia, and Hyperhidrosis. Med Clin North Am.vol. 99(6),pp.1195-1211,2016.

[3] T. Phillips, W .Slomiany, R .Allison. Hair Loss: Common Causes and Treatment. Am Fam Physician .vol.96(6),pp.371-378,2017.

[4] A.Rebora. Proposing a simpler classification of telogen effluvium. Skin appendage disorders.vol.2(1-2),pp.35-38, 2016.

[5] C.Grover, A.Khurana. Telogen effluvium. Indian Journal of Dermatology, Venereol Leprol .vol.79(5) ,pp.591-603,2013.

[6] R.Trueb. Serum Biotin Levels in Women Complaining of Hair Loss. Int $\mathbf{J}$ Trichol.vol. 8(2),pp.73-77,2016. 
[7] K.Seymons, A.De Moor, H.De Raeve, J.Lambert. Dermatologic signs of biotin deficiency leading to the diagnosis of multiple carboxylase deficiency. Pediat Dermatol.vol. 21(3),pp. 231-235,2004.

[8] D.Patel, S.Swink, L.Castelo-Soccio. The Use of Biotin for Hair Loss. Skin Appendage Disord. .vol. 3(3),pp.166-169,2017.

[9] J.Stoehr, J.Choi, M.Colavincenzo, S.Vanderweil. Off-Label Use of Topical Minoxidil in Alopecia: A Review. Am J Clin Dermatol.vol. 20(2),pp.237-250,2019.

[10] S.Udompanich, K.Chanprapaph, P.Suchonwanit. Hair and Scalp Changes in Cutaneous and Systemic Lupus Erythematosus. Am J Clin Dermatol.vol.19(5),pp.679-694,2018.

[11] J.Zempleni, Y.Hassan, S.Wijeratne. Biotin and biotinidase deficiency. Expert Rev Endocrinol Metab.vol.3(6),pp.715-724,2008.

[12] S.Abdel Rahman, R.Salem, J.Sabry. Biotin Deficiency in Telogen Effluvium: Fact or Fiction? J Clin Aesthet Dermatol.vol.13(3),pp. 37-40, 2020.

[13] S.Harrison, W.Bergfeld. Diffuse hair loss: Its triggers and management. Cleve Clin J Med.vol.76,pp.361-367,2009.

[14] F.El-Esawy, M.Hussein, A.Ibrahim. Serum biotin and zinc in alopecia areata. J Cosm Dermatol.vol. 18(5),pp. 1546-1549, 2019.

[15] D.Daulatabad, A.Singal, C.Grover, N.Chhillar. Prospective analytical study evaluating serum biotin, vitamin b12, and folic acid in patients with premature canities. Int $\mathbf{J}$ Trichol.vol. 9(1),pp. $19-24,2017$.

[16] H.Almohanna, A.Ahmed, J.Tsatalis, A.Tosti. The role of vitamins and minerals in hair loss. Dermatol Therapy.vol. 9(1),pp. 51-70, 2019. 